\title{
Beitrag zur Ermittlung der Gesamtverfügbarkeit in Fließproduktionssystemen
}

\author{
Dipl.-Ing. Ayman Al Khateeb \\ Technische Universität IImenau \\ Fachgebiet Fabrikbetrieb
}

\begin{abstract}
Zur Realisierung eines gewünschten Produktionsdurchsatzes können im Prinzip viele Strukturalternativen von Fließproduktionssystemen zur Verfügung stehen, die durch die Variierung von Stationstaktzeiten und parallelen identischen Fließlinien ermöglicht werden. Im Fachgebiet Fabrikbetrieb der TU IImenau wurden diese parallelen geleichartigen Fließlinien so weiterentwickelt, dass sie durch symmetrische Puffer kombiniert werden. Da diese Strategie von größter Bedeutung ist, ist es empfehlenswert, die Gesamtverfügbarkeit von kombinierten Strukturen zu untersuchen. Dieser Beitrag stellt eine analytische Methode und die Simulation zur Berechnung von Gesamtverfügbarkeit dar. Viele Simulationsuntersuchungen werden durchgeführt, um die Gesamtverfügbarkeit, die Stationskennzahlen und das Systemverhalten zu analysieren. Im Allgemeinen beschreibt der Beitrag, wann und warum die Kombination von gleichartigen Fließlinien zur Erhöhung von Gesamtverfügbarkeit vorteilhaft ist.
\end{abstract}

\section{$1 \quad$ Einleitung}

Bevor die Methoden zur Ermittlung der Gesamtverfügbarkeit vor und nach der Kombination von Fließlinien erläutert werden, ist es sinnvoll, einen Überblick in die Gestaltung von kombinierten Fließproduktionssystemen zu geben.

\subsection{Kombinierte Fließproduktionssysteme}

Zur Erreichung einer vorgegebenen Ausbringungsmenge für ein Erzeugnis im Planungszeitraum ist die Struktur des Fließsystems zu beachten und auszuwählen. Die Strukturalternativen unterscheiden sich untereinander mit den Anzahlen der gleichartigen parallelen Fließlinien, Stationenzahlen in jeder Linie und den Taktzeiten. Die Soll-Mengenleistung in jeder Alternative ergibt sich nach [Zäp00] aus:

Soll - Mengenleistung $=\frac{\text { Arbeitszeit }}{\text { Taktzeit }} \times$ Anzahl paralleler gleichartiger Fließlinien

$\mathrm{Zu}$ kleine Stationstaktzeiten erhöhen die Stationenzahl und dadurch die Verkettungsverluste. Je größer die Anzahl gleichartiger Fließlinien, desto größer ist die Stationstaktzeit und gleichzeitig ist die Stationenzahl pro Fließlinie geringer, damit verkompliziert sich das Gesamtsystem und die technische Verfügbarkeit vermindert sich. Unter Berücksichtigung, dass bei komplexen Anlagen die Gesamtverfügbarkeit 
den Wert 98 \% nicht überschreitet (vgl. [VDI90]) und dass die Bearbeitungszeiten bspw. bei manuellen Arbeiten schwanken können, sind Puffer zwischen den Stationen vorzusehen. $\mathrm{Da}$ solche Strukturen zur Gestaltung von Fließproduktionssystemen von Bedeutung sind, werden sie im Fachgebiet Fabrikbetrieb der TU IImenau untersucht. Eine neue Strategie wurde entwickelt, in welcher die parallelen gleichartigen Fließlinien miteinander verbunden werden [ALK10]. Hieraus ergibt sich eine besonders günstige Kombination von Fließlinien. Die wesentlichen Resultate dieser Strategie lassen sich wie folgt zusammenfassen:

- Erhebliche Reduzierung der erforderlichen Pufferplätze in Höhe von mehr als $50 \%$ je nach der Anzahl der parallelen Fließlinien

- Erhöhung des Gesamtdurchsatzes

\subsection{Berechnung der Gesamtverfügbarkeit in konventioneller bzw. kombinierter Struktur}

Um die Gesamtverfügbarkeit eines Fließsystems umfassend zu untersuchen, sind die mathematische Formeln und die Simulation zu benutzen. Die VDI-Berichte bzw. Richtlinien bieten die benötigten Formeln an, die nicht nur zur Messung der Verfügbarkeiten von Einzelkomponenten sondern auch Gesamtanlagen und komplexen Materialflusssystemen nutzbar sind. Man unterscheidet zwischen elastisch und starr kombinierten Systemen (vgl. Tabelle 1). In zahlreichen Literaturquellen (z.B. [VDI90], [Wal91]) ist die Gesamtverfügbarkeit $\left(\mathrm{V}_{\mathrm{ges}}\right)$ von starren Systemen durch die folgenden Formeln berechnet worden.

Für in Reihe geschaltete Systemkomponenten gilt [VDI90]:

$$
\mathrm{V}_{\text {ges }}=\prod_{\mathrm{i}=1}^{\mathrm{n}} \mathrm{V}_{\mathrm{i}} \quad \text {;n: Anzahl der Systemkomponenten }
$$

Für parallele nicht redundante Systemkomponenten gilt:

$$
\mathrm{V}_{\mathrm{ges}}=\frac{\sum_{\mathrm{i}=1}^{\mathrm{m}} \mathrm{V}_{\mathrm{i}}}{\mathrm{m}} \quad ; \mathrm{m} \text { : Anzahl der parallelen Komponenten }
$$

Für parallele redundante Systemkomponenten gilt:

$$
V_{\text {ges }}=1-\prod_{i=1}^{m}\left(1-V_{i}\right)
$$

Zur Berechnung von elastisch verketteten Anlagen bietet das mathematische Verfahren bisher keine genaue Lösung [Mil99], da die Darstellung von Puffereinflüssen auf 
die Verfügbarkeit der Gesamtanlage durch Formeln kaum möglich ist [Mai10]. Deshalb findet man nur sehr wenige Literaturquellen, die diese Puffereinflüsse behandeln, und dann auch nur als Sonderfall. [Jan79] hat im Rahmen seiner Dissertation eine Formel zur Ermittlung der Gesamtverfügbarkeit von Systemen mit Engpasselementen entwickelt.

$V_{g e s}=\frac{1}{1+\frac{1}{t_{\text {eng }}} \sum_{i=1}^{n} t_{i}\left(\frac{1}{V_{i}}-1\right)} ; \mathrm{t}_{\text {eng }}, \mathrm{t}_{\mathrm{i}}:$ Taktzeit des Engpass- bzw. nicht Engpasselements (5)

Die Berechnung der Gesamtverfügbarkeit mit konventionellen Strukturen erfolgt durch die Berechnung des Durchschnitts der Gesamtverfügbarkeit einzelner paralleler Linien.

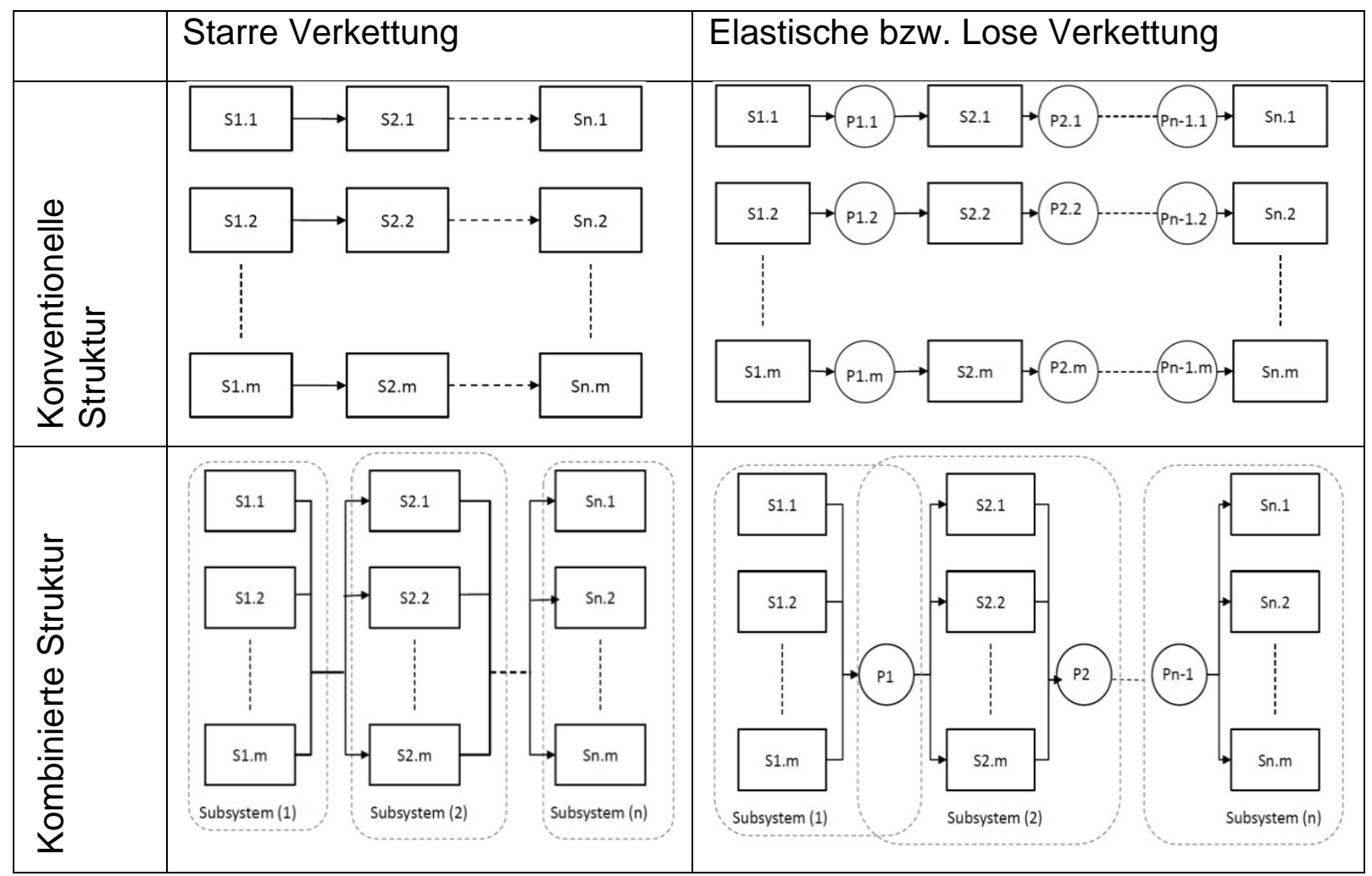

Tabelle 1: Konventionelle und kombinierte Strukturen bei starren bzw. elastischen Verkettungen

Ein kombiniertes Fließproduktionssystem umfasst eine Zusammenstellung von parallelen und in Reihe geschalteten Systemelementen. Bei kombinierten Strukturen wurde eine Methode verwendet, mit der das gesamte System in $n$ kleine Subsysteme unterteilt wird (Siehe Tabelle 1), im jeweiligen Subsystem wird die Gesamtverfügbarkeit nach den Gleichungen (3) und (4) berechnet. Da die Subsysteme in Reihe geschaltet sind, sind die Gleichungen (2) bzw. (5) für starre bzw. elastische Systeme nutzbar. Für das starr kombinierte System gilt: 
$V_{\text {ges }}=\prod_{i=1}^{k} V_{\text {sub. }} ;$; $\mathrm{k}$ Anzahl der in Reihe geschalteten Subsysteme

Für das elastisch kombinierte System gilt:

$$
V_{\text {ges }}=\frac{1}{1+\frac{1}{t_{\text {eng }}} \sum_{i=1}^{k} t_{i}\left(\frac{1}{V_{\text {sub. } i}}-1\right)}
$$

Unter Annahme, dass die Verfügbarkeit jeder Station identisch ist und unter Verwendung von Gleichungen (2)-(7) gelten die Gleichungen aus Tabelle 2.

Dabei ist darauf zu achten, dass die verwendeten Gleichungen und die Strategie zur Berechnung der Gesamtverfügbarkeit kritisch zu betrachten sind, da die gestellten Kriterien zur Entwicklung der Formel in elastischen Systemen nicht dem untersuchten Modell entsprechen können.

\begin{tabular}{|l|l|l|}
\hline & Redundant parallele Stationen & $\begin{array}{l}\text { Nicht redundant parallele Stationen } \\
\text { (gleich wie konventionelle Linien) }\end{array}$ \\
\hline $\begin{array}{l}\text { Starre } \\
\text { Systeme }\end{array}$ & $V_{\text {ges }}=\left[1-\left(1-V_{i}\right)^{m}\right]^{n}$ & $V_{\text {ges }}=V_{i}^{n}$ \\
\hline $\begin{array}{l}\text { Elastische } \\
\text { Systeme }\end{array}$ & $V_{\text {ges }}=\frac{1}{1+n\left(\frac{1}{1-\left(1-V_{i}\right)^{m}}-1\right)}$ & $V_{\text {ges }}=\frac{1}{1+n\left(\frac{1}{V_{i}}-1\right)}$ \\
\hline
\end{tabular}

Tabelle 2: Formeln zur Berechnung der Gesamtverfügbarkeit in kombinierten Systemen

Im Allgemeinen beeinflusst die produktive Zeit der Engpassstation die Gesamtverfügbarkeit und den Gesamtnutzungsgrad eines Fließsystems. [Wal91] erwähnt die folgenden Gleichungen zur Berechnung von Systemkennzahlen.

$V_{\text {ges }}=\frac{\sum_{i} T B F_{S+f, i, \text { Eng }}}{T_{B e t r, A n l}}=\frac{\sum \text { Dauer des Laufdauerabschnitts i der Engpassstation }}{\text { Betriebsdauer des Systems }}$

Oder

$V_{\text {ges }}=\frac{M T B F(\text { der Engpassstation })}{M T B F+M T T R(\text { der Engpassstation })}$ 
$N_{\text {ges }}=\frac{\sum_{i} T B F_{s+f, i, E n g}}{T_{\text {Nutz }}}=\frac{\sum \text { Dauer des Laufdauerabschnitts i der Engpassstation }}{\text { Nutzungsdauer des Systems }}$

Unter Annahme, dass während der Untersuchung die Stationen arbeiten, warten sowie gestört oder blockiert werden können, gilt das Folgende:

$$
\begin{aligned}
& N_{\text {ges }}=\frac{\text { Arbeitsanteil }}{(\text { Arbeitsanteil }+ \text { Störungsanteil }+ \text { Blockierungsanteil }+ \text { Wartungsanteil })^{1}} \\
& \rightarrow N_{\text {ges }}=\text { Arbeitsanteil }
\end{aligned}
$$

\section{Modellaufbau}

Das Simulationsmodell ist groß genug auszuwählen, um möglichst ausführliche Ergebnisse aus den Untersuchungen zu erzeugen (siehe Tabelle 1). Jede der drei parallelen gleichartigen Fließlinien besteht bei der konventionellen Struktur aus 10 Stationen und 9 Puffern, die identisch und in Reihe geschaltet sind. Entsprechend der Vielfalt von Produkten und der in der Industrie betriebenen Bearbeitungsstationen werden die Stationsparameter so angenommen, dass die mittleren Bearbeitungszeiten der Stationen $15 \mathrm{~s}$, die Abweichung $4 \mathrm{~s}$, die untere Schranke $10 \mathrm{~s}$ und die obere Schranke $20 \mathrm{~s}$ betragen. Die mittlere Reparaturzeit ist 3 min exponentiell verteilt (vgl. [Wal91]).

Die Berechnung der Gesamtverfügbarkeit in einem starren System hängt nach analytischer Methode i. Allg. von den Verfügbarkeiten seiner Komponenten ab. Im Vergleich dazu basiert sie, nach [Jan79], in elastischen Systemen zusätzlich auf den Bearbeitungszeiten der Engpass- und Nicht-Engpass-Stationen. Die Puffer sind während der Untersuchung gleich groß angenommen, und die Aufenthaltsdauer eines Erzeugnisses im jeweiligen Puffer beträgt 1 min. In der primären Untersuchungsreihe werden die Pufferanlagen jeweils mit 100 Pufferplätzen vorgesehen, um die Einflüsse der Pufferkapazitätswerte so weit wie möglich auf die Ergebnisse zu vernachlässigen. Das kombinierte Modell ist für beide Fälle (starr und elastisch) in Tabelle 1 dargestellt.

\section{$3 \quad$ Ergebnisse und Diskussion}

Am Anfang erfolgt die Untersuchung bei unterschiedlichen Verfügbarkeitswerten der Einzelstationen. Obwohl die Stationen in jeder Linie identisch mit gleicher mittlerer Bearbeitungszeit angenommen werden, gilt die letzte Station in jeder Linie als Engpass mit geringstem Arbeitsanteil, da sich die Verkettungsverluste bei Erhöhung der Stationenzahl vergrößern.

\footnotetext{
${ }^{1}$ Diese Zeitanteile summieren sich zu $100 \%$ auf. 
Je nach dem Belegungs-, Leerzustand bzw. gestörten Zustand einer der nächsten parallelen Stationen wird das Erzeugnis aus dem Puffer an eine freie Station weitergeleitet. Die Stationen können als redundant bezeichnet werden. Ist eine oder mehrere der parallelen Stationen gestört oder blockiert und die andere(n) parallele(n) Station(en) bereits besetzt, kann eine parallele Stationsanordnung nicht mehr als redundant aufgefasst werden. Diese beiden Fälle werden durch die Formeln beachtet und mit Simulationsergebnissen verglichen. Das Systemverhalten wird bei verschiedenen Verfügbarkeiten der Einzelstationen analysiert. Die Tabellen 3, 4 und 5 werden individuell betrachtet und die entsprechenden Aussagen für die jeweilige Tabelle erläutert.

Die Tabelle 3 zeigt deutlich einen Unterschied zwischen der Gesamtverfügbarkeit in den konventionellen und kombinierten Strukturen, so dass sich eine signifikante Erhöhung der Gesamtverfügbarkeit bei kombinierter Struktur ergibt.

\begin{tabular}{|c|c|c|c|c|c|c|c|c|}
\hline Stationsverfügbar- & \multicolumn{2}{|l|}{$60 \%$} & \multicolumn{2}{|l|}{$90 \%$} & \multicolumn{2}{|l|}{$95 \%$} & \multicolumn{2}{|l|}{$98 \%$} \\
\hline Struktur & Kon. & Kom. & Kon. & Kom. & Kon. & Kom. & Kon. & Kom. \\
\hline $\begin{array}{l}\text { Gesamtverfügbar- } \\
\text { keit der starren } \\
\text { Struktur (\%) }\end{array}$ & $\overline{0,6}$ & 51,61 & 34,86 & 99 & 59,87 & 99,87 & 81,70 & 99,99 \\
\hline $\begin{array}{l}\text { Gesamtverfügbar- } \\
\text { keit der } \\
\text { elastischen } \\
\text { Struktur (\%) }\end{array}$ & 13,04 & 59,39 & 47,36 & 99 & 65,51 & 99,87 & 83,05 & 99,99 \\
\hline
\end{tabular}

Tabelle 3: Analytische Ergebnisse der starr bzw. elastisch verketteten Strukturen (Kon.= Konventionelle, Kom.= Kombinierte)

\begin{tabular}{|l||l|l||l|l||l|l||l|l|}
\hline $\begin{array}{l}\text { Stationsver- } \\
\text { fügbarkeit }\end{array}$ & \multicolumn{3}{|l||}{$60 \%$ \% } & \multicolumn{2}{|l||}{$90 \%$} & \multicolumn{2}{l||}{90} & \multicolumn{2}{l|}{$\%$} \\
\hline Struktur & Kon. & Kom. & Kon. & Kom. & Kon. & Kom. & Kon. & Kom. \\
\hline $\begin{array}{l}\text { Gesamtver- } \\
\text { fügbarkeit \% }\end{array}$ & $\begin{array}{l}11,2 \\
2\end{array}$ & 37,35 & 76,87 & 85,5 & 91,21 & 93,17 & 97.34 & 97,57 \\
\hline $\begin{array}{l}\text { Gesamtnutz- } \\
\text { ungsgrad \% }\end{array}$ & 5,09 & 23,20 & 33,55 & 58,98 & 52,44 & 68,52 & 68.89 & 77,22 \\
\hline $\begin{array}{l}\text { Ausbringungs- } \\
\text { menge (Stk) }\end{array}$ & 4385 & 20046 & 28977 & 50947 & $\begin{array}{l}4524 \\
9\end{array}$ & 59191 & $\begin{array}{l}5950 \\
4\end{array}$ & 66729 \\
\hline
\end{tabular}

Tabelle 4: Simulationsergebnisse der starr verketteten Struktur 


\begin{tabular}{|c|c|c|c|c|c|c|c|c|}
\hline Stationsver- & \multicolumn{2}{|l|}{$60 \%$} & \multicolumn{2}{|l|}{$90 \%$} & \multicolumn{2}{|l|}{$95 \%$} & \multicolumn{2}{|l|}{$98 \%$} \\
\hline Struktur & Kon. & Kom. & Kon. & Kom. & Kon. & Kom. & Kon. & Kom. \\
\hline $\begin{array}{l}\text { Gesamtver- } \\
\text { fügbarkeit \% }\end{array}$ & $\begin{array}{l}58,1 \\
9\end{array}$ & 58,26 & 89,51 & 89,31 & 95.03 & 94.75 & 98,07 & 98,07 \\
\hline $\begin{array}{l}\text { Gesamtnutz- } \\
\text { ungsgrad \% }\end{array}$ & $\begin{array}{l}53,6 \\
6\end{array}$ & 54,02 & 85,67 & 86.28 & 92,09 & 92,61 & 96,08 & 96,42 \\
\hline $\begin{array}{l}\text { Ausbringungs- } \\
\text { menge (Stk) }\end{array}$ & $\begin{array}{l}4634 \\
0\end{array}$ & 46681 & 73982 & 74568 & $\begin{array}{l}7957 \\
1\end{array}$ & 79992 & $\begin{array}{l}8302 \\
2\end{array}$ & 83295 \\
\hline
\end{tabular}

Tabelle 5: Simulationsergebnisse der elastisch verketteten Struktur

Die Werte dieser Erhöhung unterscheiden sich deutlich je nach den untersuchten Verfügbarkeiten der Einzelstationen. Weiterhin ist bei kombinierter Struktur mit größerer Stationsverfügbarkeit sichtbar, dass die Gesamtverfügbarkeit größer als die Verfügbarkeit der Einzelstationen bzw. Engpassstationen ist. Dies ist nur mit redundanten Stationen möglich. Diesen Ergebnissen kann u. U. nicht vertraut werden, weil ein signifikanter Unterschied zwischen den Gesamtverfügbarkeiten in der konventionellen und kombinierten Struktur auftritt (z.B. bei $V_{i}=60 \%$ ). Es stellt sich die Frage, in wieweit die benutzten Gleichungen bzw. die Berechnungsmethode überzeugend sind.

Demgegenüber weisen die Simulationsergebnisse in Tabelle 4 darauf hin, dass insbesondere bei geringen Verfügbarkeitswerten die Kombination der Fließlinien von Bedeutung ist, um die Gesamtverfügbarkeit zu erhöhen. Darüber hinaus lässt sich bemerken, dass der Gesamtnutzungsgrad die bessere Variante zur Schätzung der resultierenden Ausbringungsmenge und zur Beschreibung des Verhaltens verketteter Fließsysteme ist als die Gesamtverfügbarkeit.

Die Resultate in Tabelle 5 sind bemerkenswert; es besteht fast kein Unterschied zwischen der Gesamtverfügbarkeit bzw. dem Gesamtnutzungsgrad bei den beiden Strukturen. Eine kleine Verbesserung des Gesamtnutzungsgrades verursacht eine geringe Erhöhung der Ausbringungsmenge.

Die in den obigen Tabellen dargestellten Resultate bestätigen, dass sich nur die durch Simulation gewonnenen Ergebnisse bei den untersuchten Strukturen als tauglich erwiesen haben. Die analytischen Ergebnisse sind fragwürdig.

\section{$4 \quad$ Weitere Untersuchungen}

Anhand der dargestellten Ergebnisse aus Abschnitt 3 wurden weitere Simulationsreihen bei starren Strukturen durchgeführt, die mehrere Verfügbarkeitswerte der Einzelstationen und verschiedene Größen der in Reihe geschalteten Subsysteme betrachten. Generell stellen die Tabellen 6 und 7 eine signifikante Verbesserung der Gesamtverfügbarkeit bei kombinierter Struktur dar. 


\begin{tabular}{|c|c|c|c|c|c|c|c|c|c|c|}
\hline \multicolumn{2}{|c|}{$\begin{array}{l}\text { Stationsver- } \\
\text { fügbarkeit } V_{i}\end{array}$} & $60 \%$ & $65 \%$ & $70 \%$ & $75 \%$ & $80 \%$ & $85 \%$ & $90 \%$ & $95 \%$ & $98 \%$ \\
\hline \multirow{9}{*}{ 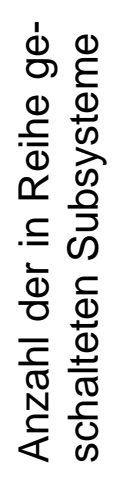 } & 2 & 6,52 & 2,3 & 9,62 & 67,24 & 75,85 & 81,53 & 87,52 & 93,74 & 98,08 \\
\hline & 3 & 34,61 & 42,7 & 51,18 & 60,8 & 71,04 & 77,16 & 85,75 & 93,38 & 97,93 \\
\hline & 4 & 27 & 34,3 & 44,02 & 53,45 & 64,3 & 74 & 66 & 32 & 71 \\
\hline & 5 & 19,51 & 28,2 & 37,9 & 48,63 & 61,64 & 73,09 & 83,11 & 93,11 & 97,63 \\
\hline & 6 & 16,89 & 22,8 & 32,87 & 42,77 & 56,75 & 69,43 & 82,18 & 92,66 & 97,49 \\
\hline & 7 & 14,07 & 19,1 & 28,21 & 38,24 & 53,22 & 68,14 & 80,16 & 92,72 & 97,41 \\
\hline & 8 & 12,59 & 17,5 & 24,66 & 35,59 & 48,72 & 66,24 & 78,91 & 92,4 & 97,23 \\
\hline & 9 & 12,13 & 16,5 & 22,41 & 33,07 & 44,7 & 60,67 & 77,6 & 91,61 & 97,11 \\
\hline & 10 & 11,31 & 15,6 & 22,05 & 30 & 44,67 & 60,16 & 75,95 & 91,59 & 97,25 \\
\hline
\end{tabular}

Tabelle 6: Simulationsergebnisse bei konventionell starrer Struktur

\begin{tabular}{|c|c|c|c|c|c|c|c|c|c|c|}
\hline \multicolumn{2}{|c|}{$\begin{array}{l}\text { Stations- } \\
\text { verfügbar- } \\
\text { keit }\end{array}$} & $60 \%$ & $65 \%$ & $70 \%$ & $75 \%$ & $80 \%$ & $85 \%$ & $90 \%$ & $95 \%$ & $98 \%$ \\
\hline \multirow{9}{*}{ 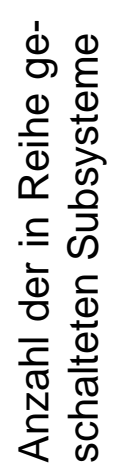 } & 2 & 51,1 & 58,3 & 63,98 & 70,82 & 76,27 & 82,58 & 88,81 & 94,31 & 97,83 \\
\hline & 3 & 47,64 & 54,9 & 60,49 & 67,67 & 74,56 & 81,03 & 88,26 & 94,49 & 97,73 \\
\hline & 4 & 44,32 & 50,8 & 57,67 & 65,55 & 72,19 & 80,34 & 87,17 & 94,33 & 97,67 \\
\hline & 5 & 42,48 & 49,2 & 57,1 & 66,22 & 71,61 & 78,81 & 87,01 & 93,5 & 98,02 \\
\hline & 6 & 40,55 & 47,3 & 55,21 & 62,52 & 71,52 & 77,96 & 85,46 & 93,67 & 97,2 \\
\hline & 7 & 38,6 & 47,1 & 53,86 & 61,33 & 70,34 & 78,53 & 86,36 & 93,01 & 97,68 \\
\hline & 8 & 38,4 & 45,5 & 52,42 & 60,59 & 69,52 & 77,83 & 85,14 & 93,25 & 97,36 \\
\hline & 9 & 37,51 & 44,6 & 52,47 & 60,56 & 68,93 & 77,09 & 86,34 & 93,44 & 97,38 \\
\hline & 10 & 35,9 & 43,7 & 52,59 & 60,88 & 68,75 & 77,34 & 86,05 & 93,46 & 97,64 \\
\hline
\end{tabular}

Tabelle 7: Simulationsergebnisse bei kombinierter starrer Struktur

Die Verfügbarkeitsgewinne sind deutlich in der linken und unteren Seite der Tabellen erkennbar und reduzieren sich ständig bei Vergrößerung der Verfügbarkeit der Einzelstationen. Im Bereich von $V_{i}=95 \%$ bzw. $98 \%$ verkleinert sich der Verfügbarkeitsgewinn bis auf ca. $2 \%$, obwohl die Anzahl der in Reihe geschalteten Subsysteme 10 ist.

Damit sich diese Ergebnisse erläutern lassen, sind die Stationszeitanteile in den beiden Strukturen darzustellen bzw. zu vergleichen. Da die letzte Station in der jeweiligen untersuchten Fließlinie nicht blockiert werden kann, wird das Verhalten der vorletzten Station analysiert (S9.1, S9.2 und S9.3 entsprechen konventioneller Struktur. S9.4, S9.5 und S9.6 entsprechen kombinierter Struktur). Betrachtet man die Abbildungen 1 und 2, so wird erkennbar, dass sich die Verkettungsverluste in Form von Wartezeiten als Hauptunterschied zwischen dem Stationsverhalten in den beiden Strukturen ausprägen.

Zudem ist zu sehen, dass die Reduzierung der Warte- bzw. Blockierzeitanteile bei den Stationen S9.1, S9.2 und S9.3 den addierten Arbeitsanteilen der Stationen S9.4, S9.5 und S9.6 beinahe gleichen. Das heißt, die Stationen in kombinierten Linien 
können teilweise als redundant gelten. Die Redundanz erfolgt, wenn drei Bedingungen gleichzeitig erfüllt sind:

- Mindestens eine der drei symmetrischen Stationen ist „hungernd“.

- Eine der drei symmetrischen Stationen ist gestört.

- Der Zugangspuffer ist mindestens mit einem Erzeugnis belegt.

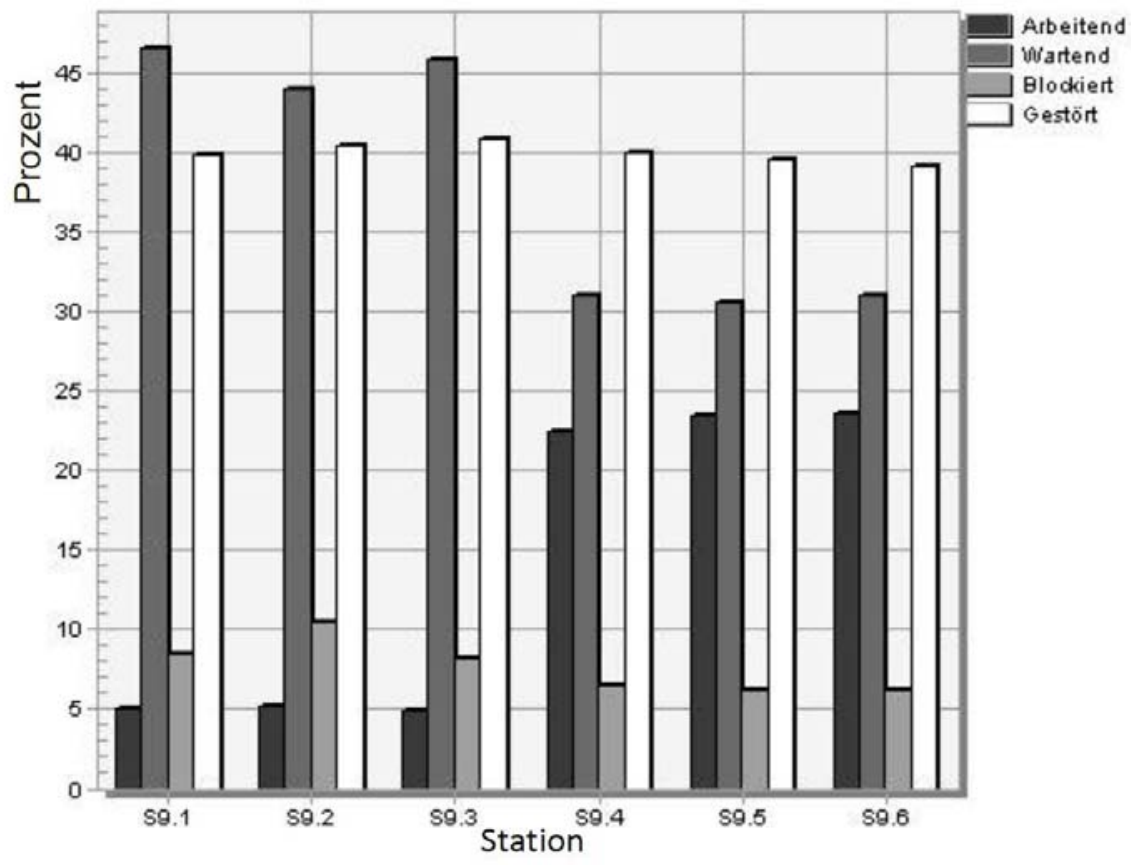

Abbildung 1: Aufteilung der Stationszeitanteile bei starrer Verkettung und $V_{i}=60 \%$

Bei der elastischen Verkettung spielen die Puffer eine signifikante Rolle, um die Verkettungsverluste zu reduzieren. Das beschränkt die Stationsfunktionen als redundant in der kombinierten elastischen Struktur und verursacht, dass die Verfügbarkeitsgewinne in kombinierten Fließsystemen gering sind. 


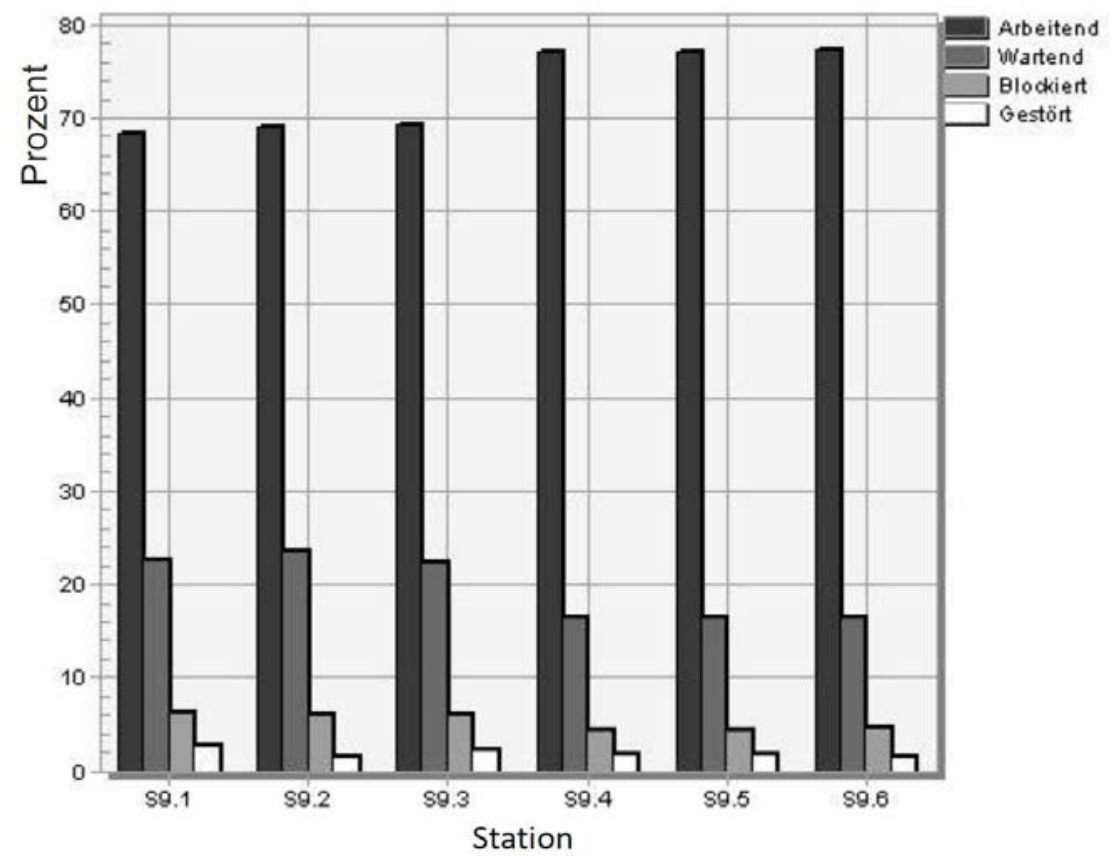

Abbildung 2: Aufteilung der Stationszeitanteile bei starrer Verkettung und $\mathrm{V}_{\mathrm{i}}=\mathbf{9 8} \%$

\subsection{Untersuchung der Puffereinflüsse auf die Gesamtverfügbarkeit}

Unter der Annahme, dass Pufferkapazitäten in der Höhe von 100 Plätzen in der Praxis nicht realisierbar sind, werden weitere Simulationsuntersuchungen unter Variation der Puffergröße durchgeführt. Es ist zu beachten, dass sich bei geringeren Pufferkapazitäten geringere Pufferinhalte ergeben. An dieser Stelle haben die erforderlichen Aufenthaltsdauern von Erzeugnissen in den Puffern signifikante Einflüsse auf die Gesamtverfügbarkeit. Deshalb wird untergestellt, dass diese Zeitwerte gleich null sind. Die Abbildung 3 verdeutlicht, dass die Erhöhung der Pufferkapazitäten bei kleinen Verfügbarkeitswerten der Einzelstationen zur Vergrößerung der Gesamtverfügbarkeit führt.

Die bisherigen gewonnenen Resultate lassen sich zusammenfassend in der Abbildung 4 darstellen.

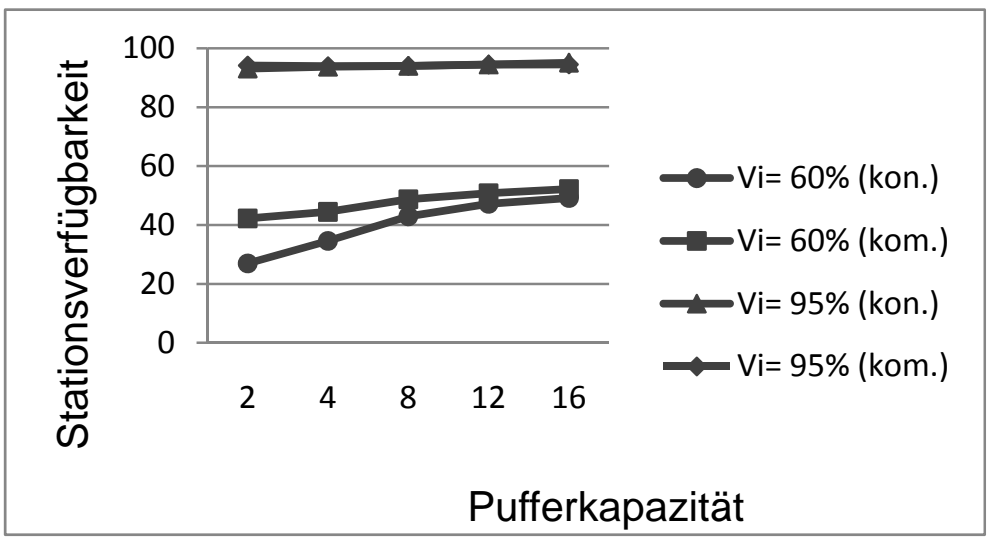

Abbildung 3: Puffereinflüsse auf die Gesamtverfügbarkeit 


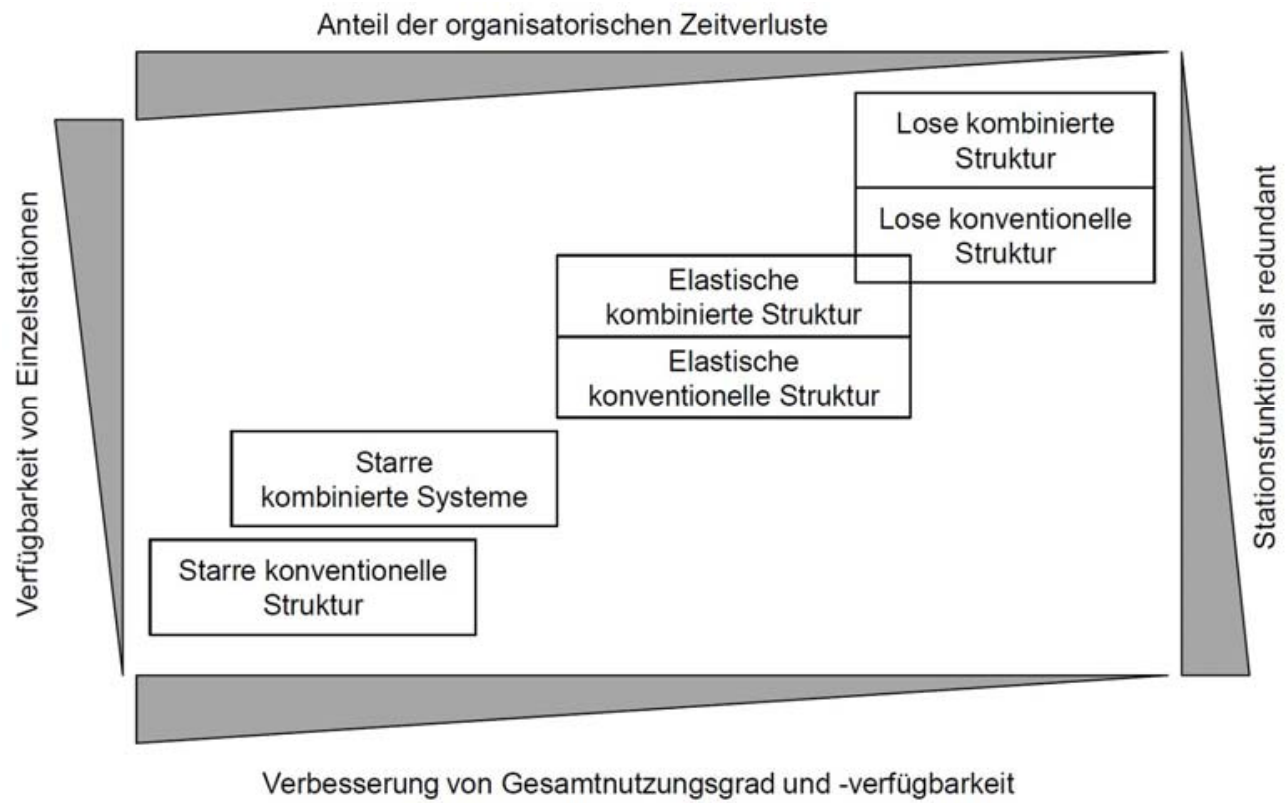

Abbildung 4: Einflüsse der Stationskennzahlen und Verkettungsarten auf den Gesamtnutzungsgrad und -verfügbarkeit in konventionellen und kombinierten Strukturen

\subsection{Addieren von einer redundanten Fließlinie}

Anhand der oben besprochenen Simulationsreihe wurde erläutert, wann und wie die Bearbeitungsstationen in der kombinierten Struktur als redundant funktionieren. In diesem Abschnitt wird eine extra redundante Linie zum Model in der kombinierten Version addiert bzw. untersucht. Die erste Station in der redundanten Linie darf nur belegt werden, wenn sie hungernd ist und wenn eine der symmetrischen Stationen gestört ist. Die Untersuchung erfolgt bei konkreten Stationsverfügbarkeiten $\left(V_{i}=\right.$ $60 \%)$ und mit der Variation der Stationsverfügbarkeit $V_{r}$ in der redundanten Linie. Die Tabelle 9 stellt die Simulationsergebnisse dar.

\begin{tabular}{|l|l|l|l|l|l|}
\hline $\begin{array}{l}\text { Stationsverfügbarkeit in der } \\
\text { redundanten Linie }\left(\mathrm{V}_{\mathrm{r}}\right)\end{array}$ & $\mathrm{V} 60 \%$ & $\mathrm{~V} 70 \%$ & $\mathrm{~V} 80 \%$ & $\mathrm{~V} 90 \%$ & V98 \% \\
\hline $\begin{array}{l}\text { Gesamtverfügbarkeit für die } \\
\text { redundante Linie }\end{array}$ & 55,18 & 64,49 & 76,91 & 87,87 & 97,43 \\
\hline $\begin{array}{l}\text { Gesamtverfügbarkeit für die } 3 \\
\text { kombinierten Linien }\left(\mathrm{V}_{\mathrm{i}}=60 \%\right)\end{array}$ & 55,82 & 54,44 & 54,41 & 52,74 & 51,53 \\
\hline $\begin{array}{l}\text { Gesamtverfügbarkeit für das } \\
\text { ganze System }\end{array}$ & 55,66 & 56,95 & 60,04 & 61,52 & 63,01 \\
\hline
\end{tabular}

Tabelle 8: Einflüsse der Addition einer redundanten Linie

Die Gesamtverfügbarkeit erhöht sich i. Allg. bei der Erhöhung des Werts $V_{r}$. Der Verfügbarkeitsgewinn $(55,66-63,1 \%)$ ist aber relativ gering, da die untersuchten Verfügbarkeitswerte $V_{i}$ klein sind. Es ist anzumerken, dass die Erhöhung des Werts $V_{r}$ mit einer Verkleinerung der Gesamtverfügbarkeit für die 3 kombinierten Linien zugleich einhergeht. 


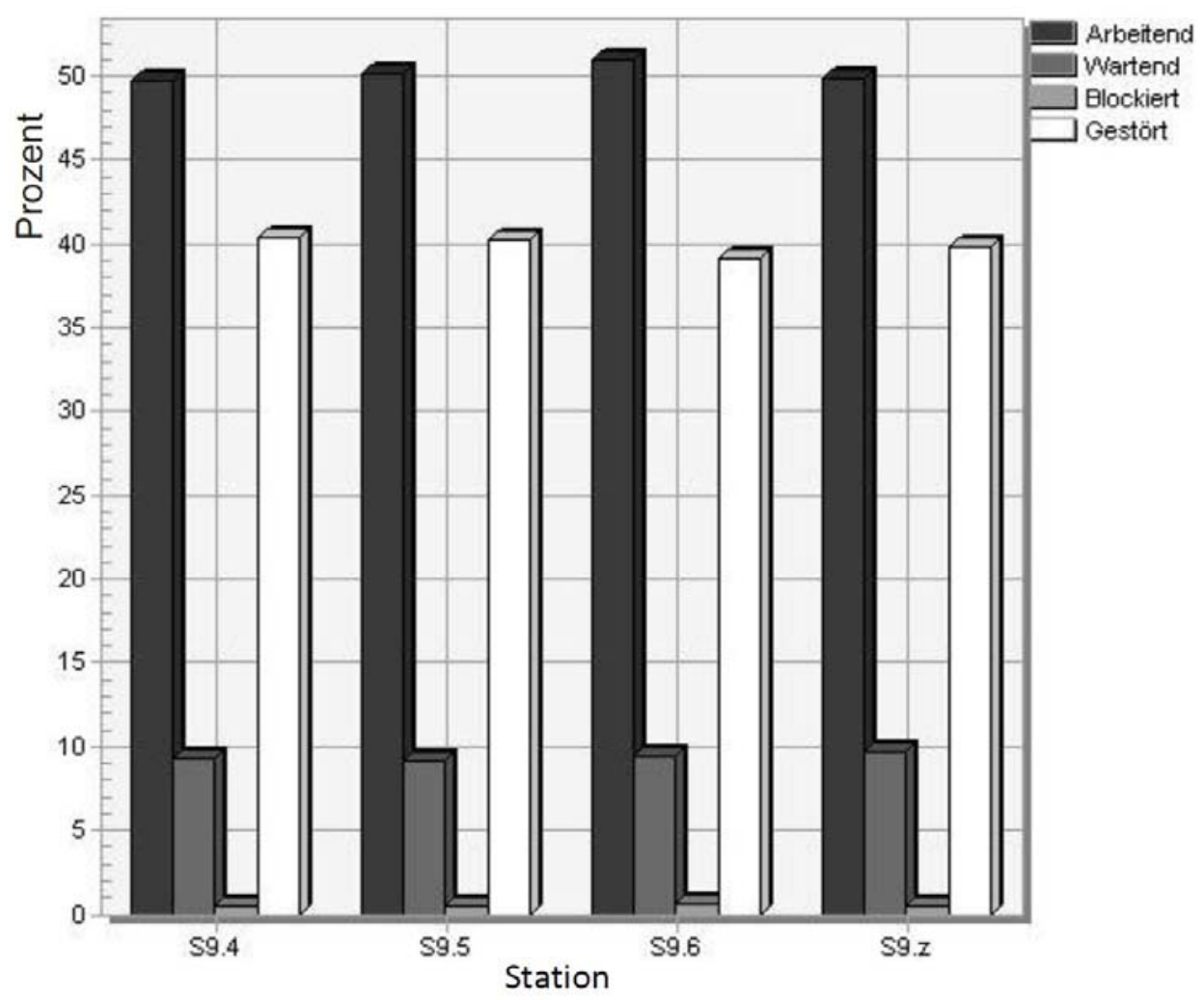

Abbildung 5: Aufteilung der Stationszeitanteile bei starrer Verkettung, $V_{i}=60 \%$ und $V_{r}=60 \%$ (S9.z ist die vorletzte Station in der redundanten Linie)

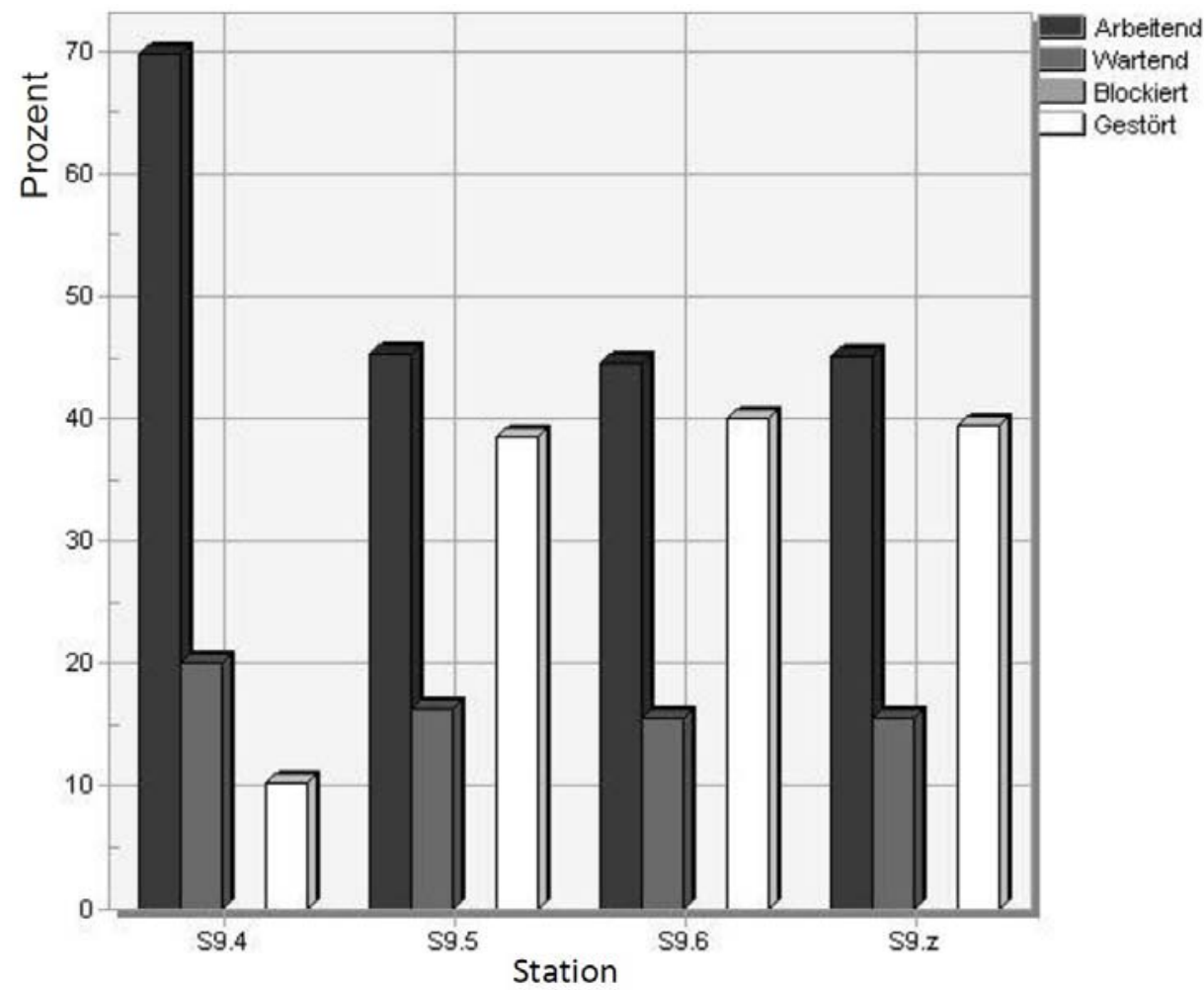

Abbildung 6: Aufteilung der Stationszeitanteile bei starrer Verkettung, $V_{i}=60 \%$ und $V_{r}=90 \%$

Ein Vergleich zwischen den Abbildungen 1, 5 und 6 zeigt, warum sich die Verfügbarkeit in der dritten Zeile reduziert. Der Grund dafür liegt in Steigerung der 
Wartezeitanteile bei den Einzelstationen, da sich die Zugangspuffer schneller entleeren.

\section{$5 \quad$ Zusammenfassung}

Dieser Beitrag hat die Gesamtverfügbarkeit von komplizierten Produktionssystemen ausführlich analysiert und wichtige Einflussfaktoren zur Erhöhung ihrer Verfügbarkeit anschaulich dargestellt. Die Kombination von Fließlinien verkompliziert das resultierende Gesamtsystem und erhöht gleichzeitig seine Gesamtverfügbarkeit bzw. seinen -nutzungsgrad. Die verwendeten Gleichungen zur Berechnung von Gesamtverfügbarkeit berücksichtigen nicht die Redundanzfunktion in kombinierten Fließsystemen. Mit Unterstützung der Simulation kann die Gesamtverfügbarkeit relativ präzise analysiert und berechnet werden. Weiterhin hilft die Untersuchung der Stationszeitanteile gute Entscheidungen zu treffen, z. B. Addition von redundanten Stationen und wirtschaftliche Erhöhung von Puffergrößen. Die Simulationsergebnisse weisen darauf hin, dass der Verfügbarkeitsgewinn bei kombinierter Struktur umso höher ist, je höher die Verkettungsverluste in konventioneller Struktur sind.

\section{Literatur}

[AIK10] Al Khateeb, A.: Kombinierte Fertigungslinien als neue Strategie in flexiblen Fertigungssystemen, Logistics Journal, 2010

[Jan79] Janisch, H.-W.: Optimierung der Puffer bei elastisch verketteten Fertigungssystemen

[Mai10] Maier, M. M.: Die Verfügbarkeit intralogistischer Anlagen - Teil II: Die Entwicklung eines Kennwerts, F+H Fördern und Heben, Zeitschrift für Materialfluss und Warenwirtschaft, 2010, Nr. 3, S. 72-75

[Mil99] Milberg, J.; Reinhardt, G.: Seminarberichte, Produktivität und Verfügbarkeit ... durch Kooperation steigern, München: Herbert Utz Verlag GMBH 1999

[Wal91] Walenda, H.: Analyse des Betriebsverhaltens elastisch verketteter automatischer Montageanlagen, Düsseldorf: VDI-Verlag GmbH, 1991

[VDI90] VDI Berichte 833: Verfügbarkeit von Materialfluss-Systemen, Düsseldorf: VDI-Verlag GmbH, 1990

[Zäp00] Zäpfel, G.: Taktisches Produktions-Management, München: Oldenbourg Wissenschaftsverlag $\mathrm{GmbH}, 2000$ 